\title{
Controlled partial embedding of carbon nanotubes within flexible transparent layers
}

\author{
Elijah B Sansom ${ }^{1,3}$, Derek Rinderknecht ${ }^{1}$ and Morteza Gharib ${ }^{1,2}$ \\ ${ }^{1}$ Option of Bioengineering, California Institute of Technology, 1200 E California Boulevard, \\ Pasadena, CA 91125, USA \\ ${ }^{2}$ Option of Aeronautics, California Institute of Technology, 1200 E California Boulevard, \\ Pasadena, CA 91125, USA \\ E-mail: elijah@caltech.edu
}

Received 21 August 2007, in final form 21 October 2007

Published 13 December 2007

Online at stacks.iop.org/Nano/19/035302

\begin{abstract}
Applications of carbon nanotubes (CNTs) like field emission displays, super-capacitors, and cell growth scaffolds can benefit from controllable embedding of the CNTs in a material such that the CNTs are anchored and protrude a desired length. We demonstrate a simple method for anchoring densely packed, vertically aligned arrays of CNTs into silicone layers using spin-coating, CNT insertion, curing, and growth substrate removal. CNT arrays of 51 and $120 \mu \mathrm{m}$ in height are anchored into silicone layers of thickness 26 and $36 \mu \mathrm{m}$, respectively. Scanning electron microscopy (SEM) and optical microscopy are used to characterize the sample morphology, a $5.5 \mathrm{~m} \mathrm{~s}^{-1}$ impinging water jet is used to apply shear stress, and a tensile test shows that the silicone layer detaches from the substrate before the CNTs are ripped from the layer. The CNTs are thus well anchored in the silicone layers. The spin-coating process gives control over layer thickness, and the method should have general applicability to various nanostructures and anchoring materials.
\end{abstract}

(Some figures in this article are in colour only in the electronic version)

A wealth of applications utilizing the properties of CNTs has been demonstrated, such as field emission displays [1, 2], enhanced surface area super-capacitors [3-5], biomimetic dry adhesives [6], flexible electronics [7], and biological cell growth and tissue engineering scaffolds [8-10]. Others, such as friction drag reducing surfaces in fluid flows [11, 12], have been simulated. For devices which aim to directly take advantage of free and accessible CNTs, such as the abovementioned examples, it is of crucial importance to be able to fabricate the CNT-containing components so that the CNTs are partially exposed and not fully embedded within other materials. CNTs have also been the subject of many studies concerned with generating composite materials with enhanced mechanical, thermal, optical, and electrical properties [13]. For the development of composite materials, it is generally not necessary that the CNTs themselves be accessible to the environment or protruding in any fashion, only that they

\footnotetext{
3 Author to whom any correspondence should be addressed.
}

interact favorably with the other components of the composite. Here we demonstrate how densely packed, vertically aligned CNTs can be simply and controllably anchored within a layer of curable elastomeric material (RTV615, GE Silicones). The four steps of the method are spin-coating a layer of uncured material to produce the desired thickness, vertical insertion of the CNTs, curing the entire assembly, and removal of the original growth substrate of the CNTs. This process provides straightforward control over the thickness of the portion of the CNT which is anchored within the anchoring material, and therefore also the thickness of the remainder which is left free and protruding. Freestanding flexible composite films of elastomer and protruding CNTs can be obtained by peeling off the films from their substrate, whereby they can be transferred to a separate surface or device component, eliminating the need for final surfaces and device components to be compatible with CNT fabrication processes. The anchoring of CNTs will improve handling in further fabrication steps and increase robustness against being accidentally scraped, blown, or rinsed 
off and removed from a functional device surface. Importantly, patterns and local configurations of the original, as-grown CNTs are preserved during this anchoring process, making it compatible with standard CNT growth patterning protocols. Inversion of the as-grown CNTs, which is inherent in our method, may have additional benefits, since it is well known that CNTs grown on a surface typically have closed ends at their tips and potentially also catalyst particles there [14]. For applications such as field emission and sensing, open ends are preferable [15], which inversion may provide.

The embedding of dispersed CNTs within polymers has been the subject of many previous studies [16-18]. It has also been demonstrated that common polymers such as polymethylmethacrylate (PMMA) and polydimethylsiloxane (PDMS) can fully wet aligned arrays of CNTs and be cured to form the matrix phase in the CNT-polymer composite $[19,20]$, where the uncured polymer material has first been deposited onto the CNTs. These findings suggest that anchored and protruding aligned CNTs could be made by using aligned CNTs pre-grown on a substrate and controlling the thickness of the polymer layer into which they are inserted, as is demonstrated here. There are other relevant previous studies involving the deposition of materials onto as-grown aligned CNTs, such as gas pyrolyzed conformal coatings of Parylene-C [21], $\mathrm{SiO}_{2}$ via chemical vapor deposition (CVD) [22], electrochemical deposition of the conducting polymers polypyrrole [23] and polyaniline [24], and polyvinylacetate [25] deposition from solution for the purposes of developing electrochemical sensors and field emission sources. Applying materials in this way usually requires etching back the coating or matrix if exposed CNTs are desired; the maximum thickness of conformal coatings can be significantly smaller than the thickness of the layer of aligned CNTs, and conformal coatings are deposited everywhere on the sample, including over the entire sidewalls of the CNTs. The deposition of a material by spin-coating in particular has also been used previously, for example PDMS [26], polystyrene-toluene solution [27], a spin-onglass [28], and a polydiene rubber [29], all spin-coated on top of an array of aligned CNTs, but this approach will not give control over protruding lengths, if indeed any protruding CNTs are produced. Additional drawbacks to spin-coating a material on top of an array of CNTs, with regard to achieving controllable protruding lengths and preserving array configurations, are that growth patterns and local configurations are likely to be lost due to the local forces between the CNTs and the spin-coated material, and the entire sidewalls of the CNTs will be coated by the spin-coated material.

The possibility of transfer of an as-grown CNT array from its growth substrate to another surface has been demonstrated previously utilizing an HF etch to first detach the CNTs [30]; however, it was not possible to retain the growth patterns of the CNTs. Transfer with inherent inversion of the CNT array and retention of growth patterns has also been demonstrated using a microwave assisted hot embossing process which partially implants the CNTs into a thermoplastic polymer [31], and in another study using the screen printing of a several micron thick silver paste into which the CNTs are inserted and then the assembly cured [32]. However, neither of these two approaches results in simple control over the depth of anchoring of the CNTs.

In contrast to previous works, the method described here requires no material removal step because the aligned CNTs are inserted only to the depth of the anchoring material layer. Simple and direct control over the anchoring depth of the CNTs is provided by the spin-coating of the anchoring material prior to CNT insertion. Retention of growth patterns is also an important feature of this method.

The CNT arrays used to demonstrate this anchoring method were grown by the well-known process of thermal CVD on iron sputtered quartz surfaces [33]. This growth process as used here results in densely packed arrays of multiwalled carbon nanotubes oriented perpendicular to the growth surface wherever catalyst material is present, with the height of the array on a given sample being quite uniform. Alignment is good at larger length scales, but there is entanglement present at the nanoscale. The typical carbon nanotube diameter is about $20 \mathrm{~nm}$ and the typical inter-nanotube spacing is about $50-100 \mathrm{~nm}$. Depending on growth conditions such as growth time, feedgas flowrate (and therefore average flow velocity at the sample surface) and composition, system pressure, and thickness of the pre-deposited catalyst layer, array heights can be obtained in a range from about $10 \mu \mathrm{m}$ for shorter growth time, for example, to over $150 \mu \mathrm{m}$ for longer growth time. Alignment and array height uniformity tends to be better for taller arrays. Because of the similarity in appearance of these aligned CNT arrays to ordinary carpets, we refer to them as 'nanocarpets'.

Thermal CVD-grown nanocarpets were fabricated following previously reported methods $[34,35]$ in a 1 -inch diameter quartz tube furnace (Lindberg/BlueM, Asheville, NC) at $725^{\circ} \mathrm{C}$ on square quartz substrates $(1 \mathrm{~cm} \times 1 \mathrm{~cm} \times 0.16 \mathrm{~cm})$ which were first sputtered with $5 \mathrm{~nm}$ of iron. The furnace and samples were brought to growth temperature under a constant flow of $500 \mathrm{sccm}$ Ar and at a pressure of 600 Torr. Beginning immediately once growth temperature was reached, the feedgas mixture of $280 \mathrm{sccm}$ ethylene and $70 \mathrm{sccm}$ hydrogen was supplied for $10 \mathrm{~min}$, keeping the total pressure constant at 600 Torr. Cool-down to ambient temperature was achieved under $500 \mathrm{sccm}$ Ar and 600 Torr pressure. For the anchoring experiments, the final heights of the two nanocarpets were 51 and $120 \mu \mathrm{m}$ as measured by scanning electron microscopy (SEM).

Two approaches were followed for anchoring a nanocarpet into an RTV (RTV615) layer. Both utilize spin-coating to create uniform layers of controlled thickness. Spin-coating is a common procedure in photolithography for making thin films, whereby a substrate is held on a vacuum chuck in a horizontal orientation, the coating material is applied to the substrate, and the substrate is spun at a specified angular velocity, flinging off any excess material. In the first approach, a thin layer of uncured RTV was spin-coated onto a flat, rigid substrate-a glass slide - and the nanocarpet was then inserted vertically; the entire assembly was cured by baking, and the nanocarpet growth substrate was removed. A schematic of this 'thin layer' 

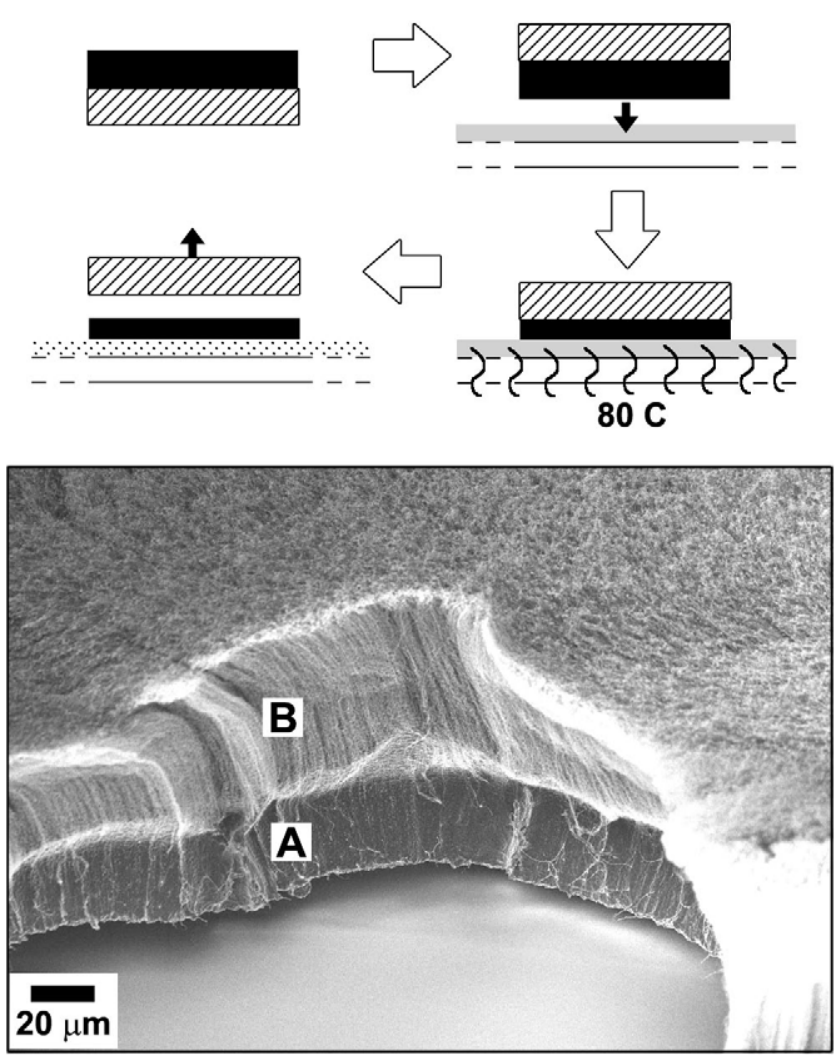

Figure 1. Nanocarpet anchored in RTV. Upper: schematic diagram showing steps in the anchoring process for this 'thin layer' approach - an as-grown nanocarpet is inserted into a spin-coated layer of uncured RTV on top of a glass slide, cured by baking at $80^{\circ} \mathrm{C}$ for over $1 \mathrm{~h}$, and the growth substrate is physically removed using tweezers, leaving the nanocarpet firmly anchored within the cured RTV layer. Lower: SEM image of a cross-section of the sample, taken at a $60^{\circ}$ off-vertical tilt angle. A indicates the RTV anchoring layer, which is $30 \mu \mathrm{m}$ thick at this location when corrected for viewing perspective, and $\mathbf{B}$ indicates the protruding, RTV-free portion of the nanocarpet which is about $64 \mu \mathrm{m}$ thick at this location when corrected for perspective. The scale bar is $20 \mu \mathrm{m}$.

approach is shown in the upper portion of figure 1 . In the second approach, a relatively thick layer of RTV was first spincoated on a piece of Teflon material and cured, followed by the spin-coating of a thin layer of uncured RTV into which the nanocarpet was inserted vertically. The entire assembly was then baked again to cure the thin layer, and the nanocarpet growth substrate was removed. This 'thin-on-thick' approach is shown in the upper portion of figure 2. Because the thick layer was cured first, the nanocarpet was inserted exactly to the depth of the uncured layer which was deposited on top of the already cured layer. The fact that the two layers were of the same material, RTV, ensured good bonding at the interface between them. Cured RTV does not adhere well to Teflon, which allowed easy release of the two joined RTV layers and anchored nanocarpet.

RTV615 is a flexible, transparent, two-part addition cure elastomer composed of polydimethylsiloxane (PDMS) and cross-linking agents, and is a common material in microfluidics. Just prior to use, the two components of the uncured RTV were mixed by manual stirring at the manufacturer recommended 10:1 ratio by weight. A glass slide

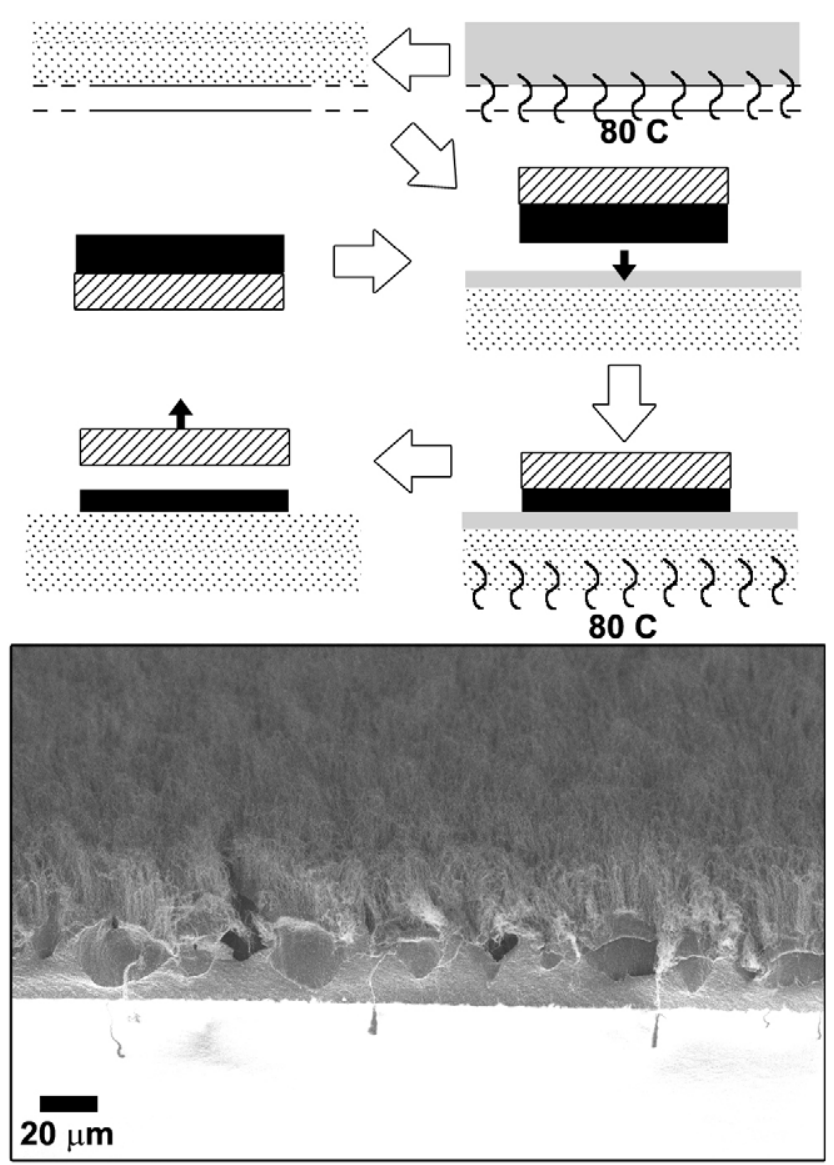

Figure 2. Nanocarpet anchored in RTV layer deposited on already cured underlayer of RTV. Upper: schematic illustrating this 'thin-on-thick' process. Lower: SEM image showing the $51 \mu \mathrm{m}$ thick nanocarpet protruding from the $26 \mu \mathrm{m}$ thick second layer and anchored within it. The second layer of RTV is easily visible compared to the thick layer underneath because the high volume fraction of conductive CNTs sufficiently disperses the charge from the incident electron beam, while the underlayer has no CNTs and is accumulating charge, resulting in low contrast. The scale bar is $20 \mu \mathrm{m}$.

was first rinsed with isopropanol (IPA) and $18.2 \mathrm{M} \Omega \mathrm{cm}$ DI water, dried with nitrogen, and secured to the bottom of a Petri dish using double-stick tape. For the thin layer approach, a small portion of the RTV was applied to the center of the secured glass slide, and spun at $2700 \mathrm{rpm}$ for 2 min, spreading the uncured RTV into a thin film on the glass slide. In the thin-on-thick layer approach, a 1/32 in thick piece of Teflon material, which had also first been IPA and DI water rinsed and nitrogen dried, was utilized to allow easy release of the RTV once the final cure was completed. A large quantity of uncured RTV mixture was spread over the Teflon surface, then baked at $80^{\circ} \mathrm{C}$. A final thin layer was then spun at $2700 \mathrm{rpm}$ for $2 \mathrm{~min}$ on top of the cured thick layer, which was still resting on the Teflon surface, followed by nanocarpet insertion into the thin layer and a final bake.

After spin-coating, a nanocarpet was carefully inserted into the spun layer of uncured RTV by inverting the nanocarpet without contacting the CNTs, and gently setting it into the layer. No additional force was applied to the growth substrate's backside. The entire assembly composed of the glass slide, 


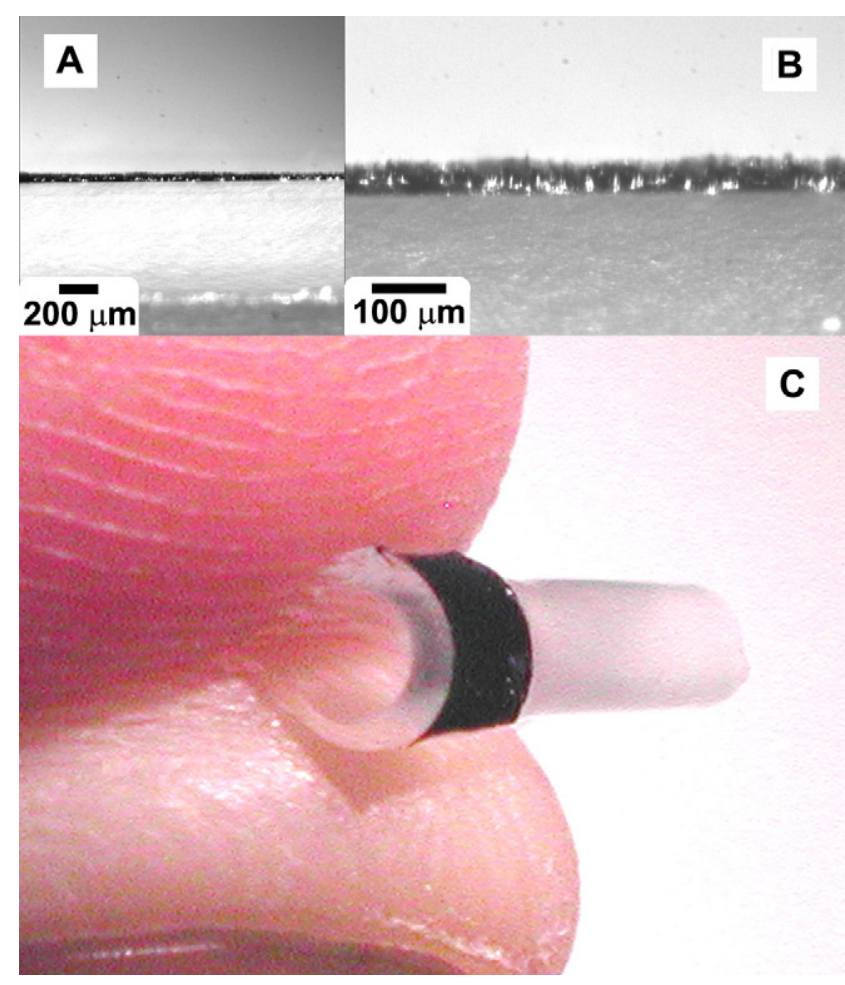

Figure 3. Images of a nanocarpet anchored within the top surface of a relatively thick transparent elastomeric film, using the 'thin-on-thick' process. (A) Low magnification optical micrograph of the cross-section, showing the transparent $630 \mu \mathrm{m}$ thick RTV layer underneath the second RTV layer with the nanocarpet anchored within it. The black layer is the nanocarpet, and the dark gray region at the bottom of the image is the sample holder for the imaging. The scale bar is $200 \mu \mathrm{m}$. (B) High magnification optical micrograph of the same sample showing the black nanocarpet protruding from the $26 \mu \mathrm{m}$ thick RTV second layer, which is joined to the thick layer underneath. The total nanocarpet thickness is about $51 \mu \mathrm{m}$. Some sections of the thin RTV layer reflect the incident light and appear white in the image. The scale bar is $100 \mu \mathrm{m}$. (C) The entire sample shown in panels (A) and (B) has been remounted in another RTV layer and is held between fingertips, demonstrating the high flexibility of the free-standing composite, transparency of the elastomeric anchoring material, and opacity of the densely packed nanocarpet. The nanocarpet is clearly anchored solely within the upper surface of the elastomer.

uncured RTV layer, nanocarpet, and growth substrate (and also the Teflon piece and double-stick tape, if used) was baked at $80^{\circ} \mathrm{C}$ overnight. Following baking, the quartz growth substrate was easily removed by gripping the sides using tweezers and lifting the substrate piece directly upward, releasing it from the nanocarpet now implanted in the cured RTV layer. This was possible because CNTs are inherently poorly adhered to their growth substrate.

For the thin layer sample, made without the Teflon piece, sections of the cured RTV layer far away from the nanocarpet implanted region were partially released and peeled up from the glass slide using a razor and tweezers, and the thickness of the RTV layer was measured to be $36 \mu \mathrm{m}( \pm 2.5 \mu \mathrm{m})$ using an outside vernier micrometer, providing an average measurement over the face area of $0.28 \mathrm{~cm}^{2}$. This thickness was later confirmed in a specific location using SEM. The final result

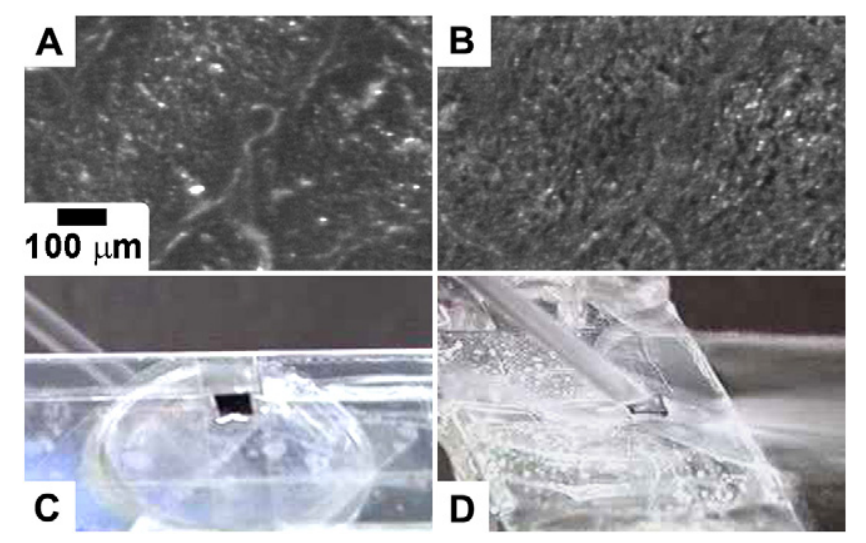

Figure 4. An anchored nanocarpet withstands a $5.5 \mathrm{~m} \mathrm{~s}^{-1}$ impinging water jet, experiencing an estimated wall shear stress of 230 dyn $\mathrm{cm}^{-2}$, comparable to the surface of a full-size ship at a similar freestream velocity. (A) Low magnification optical micrograph of the surface of the anchored nanocarpet before applying the water jet. The scale bar is $100 \mu \mathrm{m}$. (B) Same location and same scale as in panel (A), shown after applying the water jet. Constituent nanotubes have been rearranged slightly but are clearly not removed from the anchoring layer because there are no 'bald spots'. (C) Image of anchored nanocarpet before applying the jet, mounted onto a glass slide. The anchored nanocarpet is black, and is $3 \mathrm{~mm}$ on a side. (D) Anchored nanocarpet under $5.5 \mathrm{~m} \mathrm{~s}^{-1}$ water jet impinging at $45^{\circ}$.

is shown in the lower portion of figure 1. For the thin-on-thick layer sample made using the Teflon piece, the thickness of the first, thick layer of RTV was $630 \mu \mathrm{m}$, measured by optical microscopy, and the thickness of the second, thin layer of RTV into which the nanocarpet was anchored was $26 \mu \mathrm{m}$, measured by SEM. This sample is shown in the lower part of figure 2 and in figure 3.

In order to verify that nanocarpets are firmly anchored within the RTV using this method and to demonstrate the feasibility of its application in experiments on friction drag reduction, the thin-on-thick layer sample was placed in a $5.5 \mathrm{~m} \mathrm{~s}^{-1}$ water jet impinging at $45^{\circ}$. This experiment was designed to create a realistic wall shear stress on the anchored nanocarpet, similar to that expected for full-size ships at moderate speeds (10 knots). Though real watercraft may experience turbulent boundary layer flows at length-based Reynolds numbers of up to $10^{10}$, a power-law fit to measured wall shear stress data obtained from a turbulent boundary layer with length-based Reynolds numbers of up to $2.1 \times 10^{8}$ predicts a wall shear stress of between 260 and $440 \mathrm{dyn} \mathrm{cm}^{-2}$ for $6 \mathrm{~m} \mathrm{~s}^{-1}$ freestream velocity [36]. The wall shear stress caused by the $5.5 \mathrm{~m} \mathrm{~s}^{-1}$ impinging water jet was estimated to be 230 dyn $\mathrm{cm}^{-2}$ using the classical Hiemenz flow solution for a plane stagnation flow [37]. The optical micrographs in figure 4 were taken before and after the jet experiment, and along with visual observation, they clearly indicate that no regions of the nanocarpet were removed from the RTV in which they were anchored. Thus, even at realistic levels of wall shear stress, an anchored nanocarpet produced by this method remains held within the anchoring material.

A tensile test was performed to measure the strength of anchoring of CNTs within an RTV layer. Whereas the 
impinging water jet experiment demonstrated the anchored nanocarpet's ability to withstand shear stress, pulling on the nanocarpet normal to the surface can demonstrate the CNTs' behavior in tension. A sample was first carefully constructed with a nanocarpet anchored on the top and bottom in RTV layers on glass slides such that it spanned the gap between the slides. First, a $140 \mu \mathrm{m}$ tall nanocarpet was anchored in RTV in the center of a first glass slide substrate using the basic 'thin layer' form of the method. A spin speed of $7000 \mathrm{rpm}$ was selected in order to provide a very thin anchoring layer of about $20 \mu \mathrm{m}$. One important modification of the method was made. Prior to curing, the small bead of uncured RTV along the edges of the slide was carefully wiped off. Second, an RTV anchoring layer was created on a second glass slide using a spin speed of $3100 \mathrm{rpm}$ to give an anchoring layer of about $30 \mu \mathrm{m}$. The bead of RTV along the edges was removed, and the uncoated side was attached to a large flat plate of polished aluminum using superglue. Two vertical stacks made of seven glass coverslips of $160 \mu \mathrm{m}$ thickness each, giving a stack height of $1120 \mu \mathrm{m}$, were placed without gluing on the aluminum adjacent to the secured, RTV-coated glass slide. The glass slide itself was $1 \mathrm{~mm}$ thick. A drop of water was placed in the stacks of coverslips and the surface tension ensured they were in intimate contact and well adhered to each other. Any excess was dried from the surface of the two stacks. Third, the first glass slide with the anchored nanocarpet was inverted and carefully placed orthogonal to the second glass slide, resting it on the stacks of coverslips such that they supported the slide at its ends, and the anchored nanocarpet in the center was positioned over the center of the second slide. Placement of the first slide in this way inserted the protruding CNTs into the uncured RTV layer on the second slide. Fourth, the entire assembly including the aluminum plate was baked at $80^{\circ} \mathrm{C}$ for $1.5 \mathrm{~h}$ to cure the RTV. Visual inspection following baking indicated the gap region between the two glass slides was free of RTV. Fifth, a length of thin copper wire was passed through a 20 gage Luer-Lok syringe tip and knotted on one side such that the knot would fit in the syringe cavity. Sixth, the base of the Luer-Lok syringe tip was located just above the nanocarpet region, which was visible through the transparent slide and RTV, and then gently superglued to the bare, upper side of the top (first) slide. The final state of the fabrication of the sample for tensile testing is schematically shown in the upper portion of figure 5 .

In order to conduct the tensile test, the length of copper wire extending from the Luer-Lok syringe tip was passed over a pulley positioned vertically above the sample and attached to a force gage. The force was gently and steadily increased by hand until the top slide was pulled up, indicating failure within the double-anchored nanocarpet structure due to tensile stress. The peak force measured was $1.29 \mathrm{~N}$, and the test was conducted over $30 \mathrm{~s}$. The force to suspend the top slide alone was $0.08 \mathrm{~N}$. Optical microscope inspection revealed that the anchoring layer in the region of the nanocarpet had uniformly detached from the upper glass slide, leaving the entire nanocarpet attached to the bottom slide, to which it was still anchored. SEM observation confirmed this, and the nanocarpet area was measured as $0.20 \mathrm{~cm}^{2}$. The normal
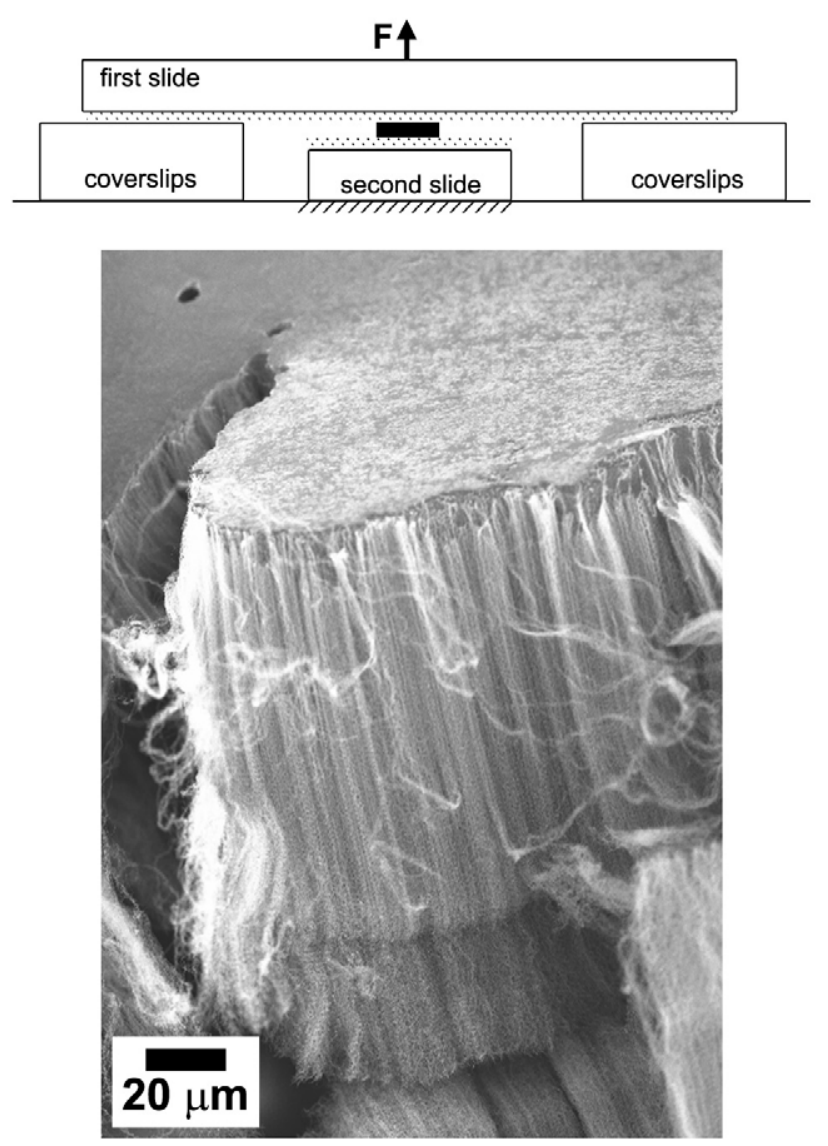

Figure 5. Fabrication and tensile testing of a double-anchored nanocarpet. Upper: side-view schematic illustrating the loading and final state of the procedure for making the double-anchored sample. The $140 \mu \mathrm{m}$ tall nanocarpet was sequentially anchored onto two glass slides placed in an orthogonal configuration. A $90 \mu \mathrm{m}$ gap was provided by stacks of coverslips supporting the top slide at its ends. Lower: SEM image following tensile testing which clearly shows that RTV is not present in the center of the nanocarpet, only at the top and bottom. This small section of nanocarpet was slightly lifted during mechanical failure of the structure. The upper surface of the nanocarpet section in the image is the upper surface of the double-anchored nanocarpet. The scale bar is $20 \mu \mathrm{m}$.

tensile stress at failure was thus $64.5 \mathrm{kPa}$ (9.35 psi). For comparison, 24 psi is the blister test measured adhesion strength of PDMS (RTV) to glass, first cleaned in piranha solution, for very minimal oxygen plasma pre-treatment [38]. The value of $64.5 \mathrm{kPa}$ measured here represents the adhesion strength of RTV to glass, not cleaned in piranha solution first, rather than the bonding strength of CNTs to RTV (anchoring strength). However, very importantly, this demonstrates that the anchoring strength of CNTs in RTV made using this method is at least $64.5 \mathrm{kPa}$, and that the CNTs are anchored more firmly in the anchoring material than the anchoring material itself is adhered to the substrate.

In summary, this paper has demonstrated the anchoring of nanocarpets within flexible, transparent RTV layers using a simple method of spin-coating and nanocarpet insertion followed by curing and removal of the nanocarpet growth substrate. Freestanding films and thick polymer layers with nanocarpets anchored only within the surface can easily be created. SEM and optical microscopy have been used to 
verify the morphology of these anchored nanocarpets, an impinging water jet experiment has confirmed their resistance to realistic shear stresses, and a tensile testing experiment has shown that the anchoring material itself detaches from the substrate before the nanocarpet is ripped from the anchoring material. The key potential features of this anchoring method are controllability over the depth of anchoring of the constituent CNTs, retention of growth patterns of vertically aligned CNTs, and scalability to sample areas larger than a few millimeters. Controllability of anchoring depth should be possible to within about $10 \mu \mathrm{m}$ or better, but will be limited by capillary effects exhibited by the uncured anchoring material. The resolution achievable in anchoring patterns of vertically aligned CNTs may also be somewhat limited by capillarity; however, even a limited degree of pattern retention combined with other features of this method represents a significant advantage over other approaches. Though RTV was used here to demonstrate nanocarpet anchoring, more specialized anchoring materials are desirable for certain applications, for example biodegradable materials for use in tissue engineering scaffolds and conductive materials for use in flexible electronics. The method demonstrated here has general applicability to these and other anchoring materials or nanostructures, since the only requirements are that the anchoring material can spread into a thin film, the anchoring material wets the nanostructures such that they are suitably embedded, and the process used for curing the anchoring material is non-damaging to the nanostructures. Many of the diverse applications foreseen for CNTs may benefit from the use of this new method and the resulting improved utility of the anchored CNTs. Easier handling and the possibility for transfer from growth surfaces to other surfaces while retaining configuration increases possible applications of CNTs, for example in the case of friction drag reducing surfaces in fluid flows. Further studies are needed to test this anchoring method with other combinations of nanostructures and anchoring materials, for example those relevant to nextgeneration photovoltaics. Further work is also necessary to conduct more detailed mechanical characterization and to determine the limits of the method's applicability.

\section{Acknowledgments}

We thank Dr Flavio Noca, Lydia Trevino, Dr Michael Bronikowski, and Bahram Valiferdowsi for assistance with the CNT growth. We thank Dr Anna Hickerson and the Keck Graduate Institute for the use of their facilities. We thank the Caltech GPS Division Analytical Facility for use of their SEM and Dr Chi Ma for assistance. We thank the National Aeronautics and Space Administration, Grant NNC05GA66G, for partial financial support of this work.

\section{References}

[1] Fan S S, Chapline M G, Franklin N R, Tombler T W, Cassell A M and Dai H J 1999 Science 283 512-4

[2] de Heer W A, Chatelain A and Ugarte D 1995 Science 270 1179-80

[3] Niu C M, Sichel E K, Hoch R, Moy D and Tennent H 1997 Appl. Phys. Lett. 70 1480-2
[4] Zhou C F, Kumar S, Doyle C D and Tour J M 2005 Chem. Mater. 171997

[5] Liu C and Cheng H M 2005 J. Phys. D: Appl. Phys. 38 231-52

[6] Zhao Y, Tong T, Delzeit L, Kashani A, Meyyappan M and Majumdar A $2006 \mathrm{~J}$. Vac. Sci. Technol. B 24 331-5

[7] Zhang M, Fang S, Zakhidov A A, Lee S B, Aliev A E, Williams C D, Atkinson K R and Baughman R H 2005 Science 309 1215-9

[8] Correa-Duarte M A, Wagner N, Rojas-Chapana J, Morsczeck C, Thie M and Giersig M 2004 Nano Lett. $42233-6$

[9] Firkowska I O M, Pazos-Perez N, Rojas-Chapana J and Giersig M 2006 Langmuir 22 5427-34

[10] MacDonald R A, Laurenzi B F, Viswanathan G, Ajayan P M and Stegemann J P 2005 J. Biomed. Mater. Res. A 74A 489-96

[11] Walther J H, Werder T, Jaffe R L and Koumoutsakos P 2004 Phys. Rev. E 69 062201(4)

[12] Ford A N and Papavassiliou D V 2006 Ind. Eng. Chem. Res. 45 1797-804

[13] Hu Y H, Shenderova O A, Hu Z, Padgett C W and Brenner D W 2006 Rep. Prog. Phys. 69 1847-95

[14] Huang S M and Dai L M 2002 J. Phys. Chem. B 106 3543-5

[15] Patil A, Li L C, Dai L M, Casavant M and Strong K 2005 J. Soc. Inf. Disp. 13 709-18

[16] Ajayan P M, Stephan O, Colliex C and Trauth D 1994 Science $2651212-4$

[17] Wagner H D, Lourie O, Feldman Y and Tenne R 1998 Appl. Phys. Lett. 72 188-90

[18] Jin L, Bower C and Zhou O 1998 Appl. Phys. Lett. 73 1197-9

[19] Raravikar N R, Vijayaraghavan A S, Keblinski P, Schadler L S and Ajayan P M 2005 Small 1 317-20

[20] Jung Y J et al 2006 Nano Lett. 6 413-8

[21] Miserendino S, Yoo J, Cassell A and Tai Y C 2006 Nanotechnology 17 23-8

[22] Li J, Stevens R, Delzeit L, Ng H T, Cassell A, Han J and Meyyappan M 2002 Appl. Phys. Lett. 81 910-2

[23] Chen J H, Huang Z P, Wang D Z, Yang S X, Li W Z, Wen J G and Ren Z F 2001 Synth. Met. 125 289-94

[24] Gao M, Huang S M, Dai L M, Wallace G, Gao R P and Wang Z L 2000 Angew. Chem. Int. Edn 39 3664-7

[25] Wei C, Dai L M, Roy A and Tolle T B 2006 J. Am. Chem. Soc. $1281412-3$

[26] Lahiff E, Ryu C Y, Curran S, Minett A I, Blau W J and Ajayan P M 2003 Nano Lett. 3 1333-7

[27] Hinds B J, Chopra N, Rantell T, Andrews R, Gavalas V and Bachas L G 2004 Science 303 62-5

[28] Nguyen C V, Delzeit L, Cassell A M, Li J, Han J and Meyyappan M 2002 Nano Lett. 2 1079-81

[29] Li L C, Yang J B, Vaia R and Dai L M 2005 Synth. Met. $154225-8$

[30] Huang S M, Dai L M and Mau A W H 1999 J. Phys. Chem. B 103 4223-7

[31] Sunden E, Moon J K, Wong C P, King W P and Graham S 2006 J. Vac. Sci. Technol. B 24 1947-50

[32] Chiu C C, Tsai T Y and Tai N H 2006 Nanotechnology 17 2840-4

[33] Melechko A V, Merkulov V I, McKnight T E, Guillorn M A, Klein K L, Lowndes D H and Simpson M L 2005 J. Appl. Phys. 97 041301(39)

[34] Bronikowski M J, Manohara H M and Hunt B D 2006 J. Vac. Sci. Technol. A 24 1318-22

[35] Bronikowski M J 2006 Carbon 44 2822-32

[36] Sanders W C, Winkel E S, Dowling D R, Perlin M and Ceccio S L 2006 J. Fluid Mech. 552 353-80

[37] White F M 1991 Viscous Fluid Flow (New York: McGraw-Hill)

[38] Bhattacharya S, Datta A, Berg J M and Gangopadhyay S 2005 J. Microelectromech. Syst. 14 590-7 